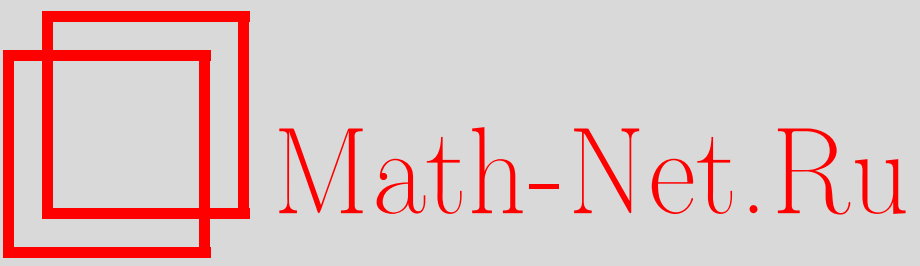

В. М. Бухштабер, Э. Г. Рис, Кольца непрерывных функций, симметрические произведения и алгебры Фробениуса, УМН, 2004, том 59, выпуск 1, 125-144

DOI: https://doi.org/10.4213/rm704

Использование Общероссийского математического портала Math-Net.Ru подразумевает, что вы прочитали и согласны с пользовательским соглашением

http://www.mathnet.ru/rus/agreement

Параметры загрузки:

IP : 54.205 .225 .156

26 апреля 2023 г., 15:16:19 


\title{
КОЛЬЦА НЕПРЕРЫВНЫХ ФУНКЦИЙ, СИММЕТРИЧЕСКИЕ ПРОИЗВЕДЕНИЯ И АЛГЕБРЫ ФРОБЕНИУСА
}

\section{В. М. БУХШТАБЕР, Е. Г. РИС}

\begin{abstract}
Приводится конструктивное доказательство классической теоремы И. М. Гельфанда и А.Н. Колмогорова (1939), характеризующей образ вычисляющего отображения компактного хаусдорфова пространства $X$ в линейном пространстве $C(X)^{*}$, двойственном кольцу непрерьвных функций $C(X)$ на $X$. Предложенный метод доказательства позволил получить более общий резултат, характеризующий образ вычисляющего отображения симметрических произведений $\operatorname{Sym}^{n}(X)$ в $C(X)^{*}$. Аналогичный резуљтат имеет место и в случае, когда $X=\mathbb{C}^{m}$. Он приводит к характеризации многообразий полисимметрических полиномов и симметрических произведений аффинных алгебраических многообразий как алгебраических подмногообразий в линейном пространстве, двойственном колцу полиномов.

Доказательство всех этих резултатов опирается на формулу, при помощи которой Фробениус в 1896 году определил высшие характеры конечных групп. Долгое время эта формула не находила дальнейших применений, но в последние десять-пятнадцать лет она неоднократно появлялась в различных независимых контекстах. Ее использовали Э. Уайлс и Р. Тейлор при изучении представлений, Х.-Ю. Хёнке и K. Джонсон, и позднее Дж. Маккай при изучении конечных групп. Она играет важную роль в наших работах по теории многозначных групп. Мы приводим описание различных свойств этой замечательной формулы. Мы применяем ее также для доказательства теоремы о структурных константах алгебр Фробениуса, которые сейчас оказались в центре внимания благодаря конструкциям, пришедшим из топологической теории поля и теории особенностей. Эта теорема развивает резултат Х.-Ю. Хёнке, опубликованный в 1958 году. В качестве следствия получено прямое замкнутое доказательство того факта, что 1-, 2- и 3-характеры регулярного представления определяют конечную группу с точностью до изоморфизма. Этот результат впервые был опубликован Х.-Ю. Хёнке и К. Джонсоном в 1992 году.
\end{abstract}

Библиограффия: 19 названий.

\section{СОДЕРЖАНИЕ}

1. Введение

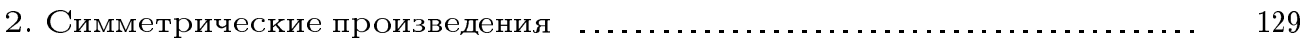

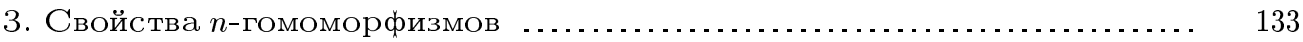

4. Алгебры Фробениуса ............................................ 137

5. Приложение А. Доказательство леммы Мэнсфильда . . . . . . . . . . . . . . 141

6. Приложение В. Алгебра полисимметрических полиномов . . . . . . . . . . 142

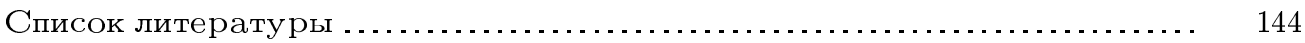

В основе настоящей статьи лежит доклад Е. Риса на конференции "Колмогоров и современная математика". Москва, 2003. 


\section{1. Введение}

В 1939 году А.Н. Колмогоров совместно с И. М. Гельфандом опубликовали работу "О кольцах непрерывных функций на топологических пространствах" (см. [11]). Центральный результат этой работы отождествляет компактное хаусдорфово пространство $X$ с пространством максимальных идеалов кольца непрерьвных функций на $X$. Все монографии и учебники, содержащие основы функционального анализа, включают теперь этот результат. В современной терминологии его можно сформулировать следуюшим образом.

Теорема 1. Пусть $X$ - компактное хаусдорфово пространство. Тогда для соответствующей топологии в пространстве непрерывных комплекснозначных функций $C(X)$ на $X$ вычисляющее отображение

$$
\mathscr{E}: X \rightarrow \operatorname{Hom}(C(X), \mathbb{C}), \quad \mathscr{E}(x) \varphi=\varphi(x),
$$

является гомеоморфизмом на множество всех кольцевых гомоморфизмов $C(X) \rightarrow \mathbb{C}$.

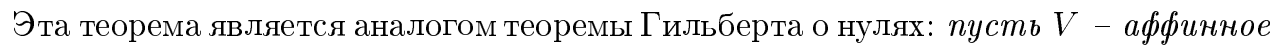
алгебраическое многообразие с координатным кольиом $A=\mathbb{C}\left[x_{1}, \ldots, x_{n}\right] / J$, где $J$ - некоторый (радикальный) идеал, задающий $V$, тогда вычисляющее отображсение

$$
\mathscr{E}: V \rightarrow \operatorname{Hom}(A, \mathbb{C})
$$

задает изоморфизм многообразия $V$ с множеством всех кольиевых гомоморфизмов.

Известные переформулировки этих теорем получаются, если заметить, что множество всех кольцевых гомоморфизмов $A \rightarrow \mathbb{C}$ нетрудно отождествить с множеством (обычно обозначаемым m-Spec $(A)$ ) всех максимальных идеалов в $A$.

Мы будем придерживаться точки зрения, что множество всех кольцевых гомоморфизмов $f: A \rightarrow \mathbb{C}$ является алгебраическим многообразием в линейном пространстве $\operatorname{Hom}(A, \mathbb{C})$, задаваемым бесконечным множеством уравнений

$$
\{f(1)=1 \text { и } f(a b)=f(a) f(b) \text { для всех } a, b \in A\},
$$

относительно координатных отображений $a: \operatorname{Hom}(A, \mathbb{C}) \rightarrow \mathbb{C}, a(f)=f(a)$. Так как мы имеем дело с линейными гомоморфизмами, то достаточно рассматривать только уравнения $f(1)=1$ и $f\left(a^{2}\right)=f(a)^{2}$, где $a$ пробегает аддитивный базис в $A$.

Стандартное доказательство теоремы Гельфанда-Колмогорова, которое можно найти в учебниках, использует приведение к следующему противоречию.

Пусть $I$ - собственньй идеал в кольце функций $C(X)$ такой, что не существует точек $x \in X$, в которых обрашаются в нуль все функщии $\varphi \in I$. Тогда для каждой точки $x \in X$ сушествует неотрицательная вещественнозначная функция $\varphi_{x} \in I$, значения которой больше 1 в некоторой окрестности $U_{x}$ точки $x$. Используя теперь компактность пространства $X$, мы можем построить вешественнозначную функцию $\varphi \in I$, значения которой больше 1 на всем $X$. Такая функция обратима, и, следовательно, в $I$ лежит постоянная функция, т.е. $I=C(X)$.

В [2] мы дали конструктивное доказательство нашей теоремы из [3], характеризующей симметрические степени $\operatorname{Sym}^{n}(X)$ как алгебраические многообразия в $C(X)^{*}$. В случае $n=1$ оно дает доказательство теоремы Гельфанда-Колмогорова, которое для данного кольцевого гомоморфизма $f: C(X) \rightarrow \mathbb{C}$ строит единственную точку 
$x \in X$ такую, что $f(\varphi)=\varphi(x)$ для всех $\varphi \in C(X)$. В многочисленной литературе на эту тему мы не нашли доказательства теоремы Гельфанда-Колмогорова, аналогичного тому, что приводится ниже.

Рассмотрим сначала случай, когда $X-$ конечное множество. Тогда $C(X) \cong \mathbb{C}^{n}$, где $n$ - число элементов в $X$. Выберем базис $\left\{\delta_{x}: x \in X\right\}$ в $C(X)$ такой, что $\delta_{x}(x)=1$, $\delta_{x}(y)=0$, если $x \neq y$. Ясно, что $1=\sum \delta_{x}$. Так как $\delta_{x}^{2}=\delta_{x}$, то для кольцевого гомоморфизма $f$ мы имеем $f\left(\delta_{x}\right)^{2}=f\left(\delta_{x}\right)$, и, следовательно, $f\left(\delta_{x}\right)=0$ или 1 . С другой стороны, $1=f(1)=\sum_{x \in X} f\left(\delta_{x}\right)$, поэтому существует единственная точка $x_{0} \in X$ такая, что $f\left(\delta_{x_{0}}\right)=1$, и используя, наконец, разложение $\varphi=\sum \varphi(x) \delta_{x}$, получаем, что $f(\varphi)=\varphi\left(x_{0}\right)$ для всех $\varphi \in C(X)$.

Покажем теперь, как распространить это рассуждение на случай, когда $X-$ компактное хаусдорфово пространство.

ОПРеДЕлЕниЕ 1 . Пусть $K \subset X$ - некоторое компактное подмножество топологического пространства $X$. Последовательность непрерьвных функций $\varphi_{r}: X \rightarrow[0,1]$ $(r \in \mathbb{N})$ назовем огораживающей для $K$, если

1) $\varphi_{r}\left(\operatorname{Supp}\left(\varphi_{r+1}\right)\right)=1$ для каждого $r \in \mathbb{N}$,

2) $\varphi_{r}(x)=1$ для всех $r \Leftrightarrow x \in K$.

Ясно, что $\varphi_{r} \varphi_{s}=\varphi_{s}$, если $s>r$.

Пример. Пусть $K$ - компактное подмножество метрического пространства $X$ с метрикой $d(\cdot, \cdot)$, тогда последовательность функций

$$
\varphi_{r}(x)= \begin{cases}0, & \text { если } d(x, K) \geqslant 1 / r, \\ 1, & \text { если } d(x, K) \leqslant 1 /(r+1), \\ (r+1)(1-r d(x, K)), & \text { если } 1 /(r+1) \leqslant d\left(x, x_{0}\right) \leqslant 1 / r,\end{cases}
$$

является огораживающей для $K$.

Определение огораживающей последовательности компакта $K$ очевидньм образом приводит к определению огораживающей сети (иначе, обобщенной последовательности) непрерывных функций $\varphi_{t}: X \rightarrow[0,1](t \in T)$, где $T$ - некоторое направленное множество. Мы оставляем читателю доказательство того, что огораживающая сеть существует для любого компакта $K$ хаусдорфова пространства $X$. Более того, чтобы не перегружать рассуждения техническими деталями, мы далее будем говорить об огораживающих последовательностях, а в конце используем то, что все полученные утверждения верны и для огораживаюших сетей.

Лемма 2. Пусть $\left\{\varphi_{r}\right\}$ - некоторая огораживающая последовательность компакта $K$. Тогда для каждого $r$ существует открытая окрестность $U_{r}$ әтого компакта, такая, что $\varphi_{r}=1$ на $U_{r}$.

ДокаЗАТЕЛЬСТво. Возьмем $U_{r}=\left\{x: \varphi_{r+1}(x)>0\right\}$. Тогда $K \subset U_{r}$ и $U_{r} \subset$ $\operatorname{Supp}\left(\varphi_{r+1}\right)$. Следовательно, $\varphi_{r}=1$ на $U_{r}$.

ЛЕмма 3. Пусть $\left\{\varphi_{r}\right\}$ - некоторая огораживающая последовательность компакта $K$ и $\psi: X \rightarrow \mathbb{R}-$ такая функция, что $\psi(x)=1$ для всех $x \in U$, где $U-$ открытая окрестность компакта K. Тогда $(1-\psi) \varphi_{r}=0$ для достаточно больших $r$. 
ДокАЗАТЕЛЬСТВО. Пусть $x \notin U$. Выберем такое $r$, что $\varphi_{r}(x)=0$, тогда $\varphi_{r}^{-1}[0,1)$ является открытой окрестностью точки $x$, замыкание которой не пересекается с $U$. Покроем компактное пространство $X \backslash U$ конечным числом таких окрестностей. Обозначим через $r_{0}$ наибольшее из $r$, соответствуюших этим окрестностям. Тогда $(1-\psi) \varphi_{r_{0}}=0$ и, следовательно, $(1-\psi) \varphi_{s}=(1-\varphi) \varphi_{r_{0}} \varphi_{s}=0$ для $s>r_{0}$.

Лемма 4. Пусть $f: C(X) \rightarrow \mathbb{C}$ - некоторьй кольцевой гомоморфизм и $\varphi_{r}-$ последовательность функиий из $C(X)$ такая, что $\varphi_{r} \varphi_{s}=\varphi_{s}$ для всех $r<s$. Тогда существует $r_{0}$ такое, что $f\left(\varphi_{r}\right)=0$ для $r \geqslant r_{0}$, либо $f\left(\varphi_{r}\right)=1$ для всех $r$.

ДокАЗАТЕЛЬСТво. Имеем $\left(f\left(\varphi_{r}\right)-1\right) f\left(\varphi_{s}\right)=0$ для всех $r<s$. Тогда если $f\left(\varphi_{r}\right) \neq 1$, то $f\left(\varphi_{s}\right)=0$ для всех $s>r$. Из этого рассуждения вытекает требуемый результат.

ОПРЕДЕЛЕНИЕ 2 . Пусть $f: C(X) \rightarrow \mathbb{C}$-некоторьй кольцевой гомоморфизм и $\left\{\varphi_{r}\right\}$ - некоторая огораживающая последовательность компакта $K \subset X$. Bесом $K$ относительно последовательности $\left\{\varphi_{r}\right\}$ назовем число $w_{f}^{\varphi}(K) \in\{0,1\}, \operatorname{paвное~} f\left(\varphi_{r}\right)$ для больших значений $r$.

ПРЕДЛОЖЕНИЕ 5. Пусть $\left\{\varphi_{r}\right\} \quad u\left\{\psi_{r}\right\}$ - две огораживающие последовательности данного компакта $K$. Тогда $w_{f}^{\varphi}(K)=w_{f}^{\psi}(K)$.

Таким образом, определен вес компакта $w_{f}(K)$, не зависящий от выбора огораживающей последовательности.

ДокАЗАТЕЛЬСТВо. Допустим, что $w_{f}^{\varphi}(K)=1$ и $w_{f}^{\psi}(K)=0$. Тогда $f\left(\varphi_{r}\right)=1$ для всех $r$, в то время как $f\left(\psi_{r}\right)=0$ для всех $r$, больших некоторого $r_{0}$. Используя лемму 3 с $\psi=\psi_{r_{0}}$, мы находим такое $m>r_{0}$, что $\psi_{r_{0}} \varphi_{m}=\varphi_{m}$. Таким образом, $f\left(1-\psi_{r_{0}}\right) f\left(\varphi_{m}\right)=0$; но $f\left(1-\psi_{r_{0}}\right)=1$, следовательно, $f\left(\varphi_{r}\right)=0$ для $r>m$. Полученное противоречие доказьвает предложение.

ОПРЕДЕЛЕНИЕ 3. Носителем кольцевого гомоморфизма $f: C(X) \rightarrow \mathbb{C}$ будем называть множество $S_{f}=\left\{x: w_{f}(x)=1\right\}$.

ПРЕДЛОЖЕНИЕ 6 . Для любого Кольцевого гомоморфизма $f$ множество $S_{f}$ состоит из одной точки.

ДокаЗАТЕльство. Допустим сначала, что $S_{f}$ содержит две различные точки $x$ и $y$. Выберем огораживающую последовательность $\left\{\varphi_{r}\right\}$ для множества $\{x, y\}$. Тогда $f\left(\varphi_{r}\right)=1$. Для достаточно больших $r$ мы можем выбрать также непрерьвные функции $\psi_{1}$ и $\psi_{2}$ такие, что $\psi_{1}(x)=1, \psi_{1}(y)=0, \psi_{2}(x)=0, \psi_{2}(y)=1$, причем $\left(\psi_{1}+\psi_{2}\right)^{-1} 1 \subset \varphi_{r}^{-1} 1$ и $\operatorname{Supp} \psi_{1} \cap \operatorname{Supp} \psi_{2}=\varnothing$. Тогда, по построению, $f\left(\psi_{1}\right)=f\left(\psi_{2}\right)=1$, т.е. $f\left(\psi_{1}+\psi_{2}\right)=2$. Пришли к противоречию.

Допустим теперь, что множество $S_{f}$ пусто. Тогда для любой точки $x \in X$ существует функция $\varphi_{x}: X \rightarrow \mathbb{R}$ такая, что $\varphi_{x}(x)=1$ и $f\left(\varphi_{x}\right)=0$. Открытые множества $\left\{y: \varphi_{x}(y)>0\right\}$ покрьвают пространство $X$. Выбрав из этого покрытия конечное подпокрытие, возьмем соответствующее ему множество функций $\varphi_{1}, \ldots, \varphi_{n}$. Тогда функщия $\Phi=\varphi_{1}+\cdots+\varphi_{n}$ не обрашается в нуль ни в одной точке из $X$, но $f(\Phi)=f\left(\varphi_{1}\right)+$ $f\left(\varphi_{2}\right)+\cdots+f\left(\varphi_{n}\right)=0$. Получаем, что, с одной стороны, $f\left(\Phi \frac{1}{\Phi}\right)=f(1)=1$, а с другой стороны, $f\left(\Phi \frac{1}{\Phi}\right)=f(\Phi) f\left(\frac{1}{\Phi}\right)=0$. Полученное противоречие доказывает предложение 6. 
Таким образом, для каждого кольцевого гомоморфизма $f: C(X) \rightarrow \mathbb{C}$ определен кольцевой гомоморфизм $\widehat{f}: C(X) \rightarrow \mathbb{C}, \widehat{f}(\varphi)=\varphi\left(x_{0}\right)$, где $S_{f}=\left\{x_{0}\right\}$. Для завершения доказательства теоремы 1 осталось показать, что $\widehat{f}=f$.

ПреДЛОЖЕнИЕ 7. Пусть $S_{f}=\left\{x_{0}\right\} \quad u \psi: X \rightarrow \mathbb{C}-$ некоторая непрерьвная функиия. Тогда $f(\psi)=\psi\left(x_{0}\right)$.

ДокАЗАТЕЛЬСтво. Пусть $\left\{\varphi_{r}\right\}$ - огораживающая последовательность точки $\left\{x_{0}\right\}$. Рассмотрим два случая.

Пусть сначала Supp $\psi \cap S_{f}=\varnothing$, тогда сушествует открытая окрестность точки $x_{0}$, на которой функция $\psi$ обрашается в нуль. Тогда $\psi \varphi_{r}=0$ для всех достаточно больших $r$ и одновременно $f\left(\varphi_{r}\right)=1$. Следовательно, $f(\psi)=0$.

Пусть теперь $x_{0} \in \operatorname{Supp} \psi$. Рассмотрим функщию $\theta_{r}=\left(\psi-\psi\left(x_{0}\right)\right) \varphi_{r}$. Имеем $\left|\theta_{r}\right| \leqslant\left|\psi-\psi\left(x_{0}\right)\right|$, и $\theta_{r}$ обрашается в нуль вне некоторой окрестности точки $x_{0}$ за счет множителя $\varphi_{r}$. Ввиду непрерывности функции $\psi$, получаем, что функция $\theta_{r}$ стремится к нулю в sup-норме, и, следовательно, $f\left(\theta_{r}\right) \rightarrow 0$ при $r \rightarrow \infty$. С другой стороны, $f\left(\theta_{r}\right)=\left(f(\psi)-\psi\left(x_{0}\right) f(1)\right) f\left(\varphi_{r}\right) \rightarrow\left(f(\psi)-\psi\left(x_{0}\right)\right) w_{f}\left(x_{0}\right)$ при достаточно больших $r$. Следовательно, $f(\psi)=\psi\left(x_{0}\right)$. Предложение 7 доказано.

\section{2. Симметрические произведения}

Напомним, что симметрической степенью пространства $X$ назьвается факторпространство

$$
\operatorname{Sym}^{n}(X)=X^{n} / S_{n}=\left\{\left(x_{1}, \ldots, x_{n}\right):\left(x_{\sigma(1)}, \ldots, x_{\sigma(n)}\right) \sim\left(x_{1}, \ldots, x_{n}\right), \sigma \in S_{n}\right\},
$$

где $S_{n}$ - группа перестановок множества из $n$ элементов.

Непрерьвные функции на $\operatorname{Sym}^{n}(X)$ - это в точности непрерывные функции $f: X^{n} \rightarrow \mathbb{C}$, инвариантные относительно перестановок координат, так назьваемые симметрические функции.

Рассмотрим аналог вычисляюшего отображения

$$
\mathscr{E}: \operatorname{Sym}^{n}(X) \rightarrow \operatorname{Hom}(C(X), \mathbb{C}), \quad \mathscr{E}\left(x_{1}, \ldots, x_{n}\right)(\varphi)=\varphi\left(x_{1}\right)+\cdots+\varphi\left(x_{n}\right) .
$$

Мы опишем образ этого отображения при помоши уравнений. Эти уравнения задаются формулами, которые впервые были использованы Г. Фробениусом [9], [10], а совсем недавно рядом авторов, включая Э. Уайлса [19], Р. Тейлора [18], Х.-Ю. Хёнке и К. Джонсона [13], Р. Рукье [17], Л. Ниссен [15]. Они играют важную роль в теории многозначных групп (см. [4]-[6]). Будем следовать подходу, развитому в [3].

Пусть $A$ - некоторая ассоциативная алгебра с единищей над полем комплексных чисел $\mathbb{C}$ и $f: A \rightarrow \mathbb{C}$ - линейное следовое отображение (т.е. $f(a b)=f(b a))$. Введем линейные отображения $\Phi_{n}(f): A^{\otimes n} \rightarrow \mathbb{C}$, полагая $\Phi_{1}(f)=f, \Phi_{2}(f)\left(a_{1} \otimes a_{2}\right)=$ $f\left(a_{1}\right) f\left(a_{2}\right)-f\left(a_{1} a_{2}\right)$ и далее по индукции

$$
\begin{aligned}
& \Phi_{n+1}(f)\left(a_{1} \otimes a_{2} \otimes \cdots \otimes a_{n+1}\right)=f\left(a_{1}\right) \Phi_{n}(f)\left(a_{2} \otimes \cdots \otimes a_{n+1}\right) \\
& \quad-\Phi_{n}(f)\left(a_{1} a_{2} \otimes a_{3} \otimes \cdots \otimes a_{n+1}\right)-\ldots-\Phi_{n}(f)\left(a_{2} \otimes a_{3} \otimes \cdots \otimes a_{1} a_{n+1}\right) .
\end{aligned}
$$

Заметим, что кольцевой гомоморфизм $f: A \rightarrow \mathbb{C}$ удовлетворяет условиям $f(1)=1$ и $\Phi_{2}(f) \equiv 0$. 
ОПРЕДЕЛЕНИЕ 4. Фробениусовыл $n$-гомоморфизмом называется линейный гомоморфизм $f: A \rightarrow \mathbb{C}$, удовлетворяющий условиям $f(1)=n$ и $\Phi_{n+1}(f) \equiv 0$.

Наш выбор названия для описанных вьше гомоморфизмов объясняется тем, что формула для определяющей рекурсии, первые примеры и важные приложения их появились в работах Фробениуса [9] и [10] для случая групповых алгебр конечных групш. Так, в [9] в наших обозначениях был получен следуюший результат.

Пусть $G$ - конечная группа и $A=\mathbb{C} G$ - ее груповая алгебра. Тогда характер $\chi: G \rightarrow \mathbb{C}$ любого $n$-мерного линейного представления групшы $G$ продолжается до линейного гомоморфизма $\chi: A \rightarrow \mathbb{C}$ такого, что $\chi(1)=n$ и $\Phi_{n+1}(\chi) \equiv 0$.

Обобшением теоремы Гельфанда-Колмогорова на случай симметрических произведений является следующий результат.

Теорема 8. Пусть $X-$ компактное хаусдорфово пространство. Тогда образ отображсния

$$
\mathscr{E}: \operatorname{Sym}^{n}(X) \rightarrow \operatorname{Hom}(C(X), \mathbb{C})
$$

представляет собой в точности подпространство всех фробениусовых $n$-гомоморфизмов, т.е. задается уравнениями $f(1)=n u \Phi_{n+1}(f) \equiv 0$.

Более детальную формулировку и конструктивное доказательство этой теоремы, использующее изложенную вьше технику огораживающих последовательностей (огораживающих сетей в случае общих компактных хаусдорфовых пространств), можно найти в [2].

Аналогично определению 4 вводится понятие фробениусовых $n$-гомоморфизмов $f: A \rightarrow B$, где $B$ - любая коммутативная алгебра. В случае, когда $B$ не имеет делителей нуля, фробениусовы $n$-гомоморфизмы обладают важньми для нас свойствами. Поэтому далее мы будем предполагать, что $B$ - область целостности. В [3] доказательство теоремы 8 получено как следствие общего результата, характеризующего фробениусовы $n$-гомоморфизмы $f: A \rightarrow B$.

Пусть $A$ - коммутативная алгебра. Обозначим через $S^{n} A$ симметрическую подалгебру в $A^{\otimes n}$. Каждьй элемент $a \in S^{n} A$ можно записать в виде

$$
a=\sum_{\sigma \in S_{n}} a_{\sigma(1)} \otimes \cdots \otimes a_{\sigma(n)}
$$

и произведение в $S^{n} A$ в виде

$$
\mathbf{a b}=\sum_{\sigma_{1}, \sigma_{2} \in S_{n}} a_{\sigma_{1}(1)} b_{\sigma_{2}(1)} \otimes \cdots \otimes a_{\sigma_{1}(n)} b_{\sigma_{2}(n)}
$$

Теорема 9. Линейное отображсение $f: A \rightarrow B$ является фробениусовым $n$-гомоморфизмом тогда и только тогда, когда $f(1)=n$ и ограничение гомоморфизма $\Phi_{n}(f): A^{\otimes n} \rightarrow B$ на $S^{n} A$ дает кольчевой гомоморфизм

$$
\frac{1}{n !} \Phi_{n}(f): S^{n} A \rightarrow B
$$

Приведем комбинаторный результат, на которьй сушественно опирается доказательство этой теоремы.

Пусть $X$ - конечное множество и $\mathscr{P}(X)$ - свободная абелева группа, порожденная множеством всех разбиений множества $X$. Напомним, что для любая перестановка $\sigma$ 
множества $X$ определяет разбиение $X$, заданное орбитами действия подгрупшы порожденной $\sigma$. Перестановки, задающие одно разбиение, имеют одинаковый знак; для данного разбиения $\pi$ обозначим через $\epsilon(\pi)$ соответствующий знак и через $n(\pi)$ - число перестановок, которые порождают $\pi$. Положим

$$
\chi(X)=\sum_{\pi} \epsilon(\pi) n(\pi) \pi \in \mathscr{P}(X)
$$

где сумма берется по всем разбиениям множества $X$.

Пусть $\pi_{1}, \pi_{2}$ - разбиения множеств $X, Y$ соответственно, тогда определено естественное разбиение $\pi_{1} \pi_{2}$ дизъюнктного объединения $X \sqcup Y$. Таким образом, определен элемент $\chi(X) \chi(Y) \in \mathscr{P}(X \sqcup Y)$.

Если $g: X \rightarrow Y$ - отображение "на" и $\pi$ - разбиение множества $Y$, то взяв прообразы частей $\pi$, получим разбиение $g^{*} \pi$; таким образом задан индуцированный гоморфизм $g^{*}: \mathscr{P}(Y) \rightarrow \mathscr{P}(X)$.

Если отображения $i_{1}: X \rightarrow Z, i_{2}: Y \rightarrow Z$ являются вложениями и $Z=i_{1}(X) \cup$ $i_{2}(Y)$, скажем, что $\left(Z, i_{1}, i_{2}\right)$ является амальгамированным облединением множеств $X$ и $Y$. Имеет место отображение "на" $q: X \sqcup Y \rightarrow Z$.

Следующий, довольно неожиданньй, чисто комбинаторньй результат был получен в [3] с использованием производящих полиномов, являющихся решением некоторого гипергеометрического дифференциального уравнения.

ЛЕмМА 10.

$$
\sum q^{*} \chi(Z)=\chi(X) \chi(Y)
$$

где сумма берется по всем различным амальгамированным обгединениям $X$ и $Y$, включая и дизбюнктное.

Ключевые моменты доказательства теоремы 9:

1. Пусть $X=\left(a_{1}, \ldots, a_{n}\right)$, где $a_{k} \in A$. Используя гомоморфизм $f: A \rightarrow B$, зададим гомоморфизм

$$
f: \mathscr{P}(X) \rightarrow B
$$

значение которого на разбиении $\pi=\left(P_{1}, \ldots, P_{k}\right) \in \mathscr{P}(X)$, где $P_{i}=\left(a_{i_{1}}, \ldots, a_{i_{q}}\right)$, равно

$$
f(\pi)=\prod_{i=1}^{k} f\left(a_{i_{1}} \cdots a_{i_{q}}\right) .
$$

Тогда

$$
f(\chi(X))=\Phi_{n}(f)\left(a_{1}, \ldots, a_{n}\right) .
$$

2. Пусть $X=\left(a_{1}, \ldots, a_{n}\right)$ и $Y=\left(b_{1}, \ldots, b_{n}\right)$ - непересекающиеся множества. Из определений получаем

$$
f(\varrho(X) \varrho(Y))=f(\varrho(X)) f(\varrho(Y)) .
$$

3. Пусть $f: A \rightarrow B$ - некоторьй $n$-гомоморфизм. На основе леммы 10 , используя, что $\Phi_{n+1}(f) \equiv 0$, получаем

$$
f(\chi(X) \chi(Y))=\sum_{\sigma \in S_{n}} f\left(\chi\left(X \sqcup_{\sigma} Y\right)\right) .
$$


Здесь $Z=X \sqcup_{\sigma} Y$ обозначает амальгамированное объединение такое, что $Z$ состоит из $n$ элементов; в этом случае отображения $i_{1}$ и $i_{2}$ являются взаимно однозначньми, и поэтому $Z$ определяется некоторой перестановкой $\sigma$.

4. Последний шаг доказательства состоит в проверке равенства

$$
\sum_{\sigma \in S_{n}} \Phi_{n}(f)\left(a_{1} b_{\sigma(1)}, \ldots, a_{n} b_{\sigma(n)}\right)=n ! \Phi_{n}(f)(\mathbf{a b})
$$

Обозначим теперь через $\Phi_{n}(A)$ множество всех фробениусовых $n$-гомоморфизмов кольца $A$ в поле комплексных чисел $\mathbb{C}$. По построению $\Phi_{n}(A)$ - алгебраическое подмногообразие в линейном пространстве $A^{*}=\operatorname{Hom}(A, \mathbb{C})$ с координатами $a: A^{*} \rightarrow \mathbb{C}$, где $a(f)=f(a)$. Положим

$$
\Phi_{n}\left(\mathbb{C}\left[u_{1}, \ldots, u_{m}\right]\right)=\Phi_{n}(m)
$$

Tеорема 11. Отображсение

$$
\begin{gathered}
\mathscr{E}: \operatorname{Sym}^{n}\left(\mathbb{C}^{m}\right) \rightarrow \operatorname{Hom}\left(\mathbb{C}\left[u_{1}, \ldots, u_{m}\right], \mathbb{C}\right), \\
\mathscr{E}\left(x_{1}, \ldots, x_{n}\right)(p)=p\left(x_{1}\right)+\cdots+p\left(x_{n}\right),
\end{gathered}
$$

задает гомеоморфизм

$$
\mathscr{E}: \operatorname{Sym}^{n}\left(\mathbb{C}^{m}\right) \rightarrow \Phi_{n}(m)
$$

На основе этого результата в приложении В мы приводим описание вложения

$$
\operatorname{Sym}^{n}\left(\mathbb{C}^{m}\right) \subset \mathbb{C}^{N}, \quad N=\left(\begin{array}{c}
n+m \\
n
\end{array}\right)-1,
$$

в контексте теории полисимметрических полиномов.

ДокАЗАТЕЛЬство. Проверка того, что вычисляюшее отображение $\mathscr{E}$ является вложением, проводится непосредственно (см. [3]). Пусть теперь $f: \mathbb{C}\left[u_{1}, \ldots, u_{m}\right] \rightarrow \mathbb{C}$ - некоторый фробениусов $n$-гомоморфизм. Тогда согласно теореме 9 отображение

$$
\frac{1}{n !} \Phi_{n}(f): S^{n}\left(\mathbb{C}\left[u_{1}, \ldots, u_{m}\right]\right) \rightarrow \mathbb{C}
$$

является кольцевым гомоморфизмом. Далее, симметрическую алгебру $S^{n}\left(\mathbb{C}\left[u_{1}\right.\right.$, $\left.\left.\ldots, u_{m}\right]\right)$ можно отождествить с алгеброй полиномиальных функций на алгебраическом многообразии $\operatorname{Sym}^{n}\left(\mathbb{C}^{m}\right)$ - алгеброй полисимметрических полиномов. Тогда согласно теореме Гильберта о нулях сушествует набор точек $\left(x_{1}, \ldots, x_{n}\right) \in \operatorname{Sym}^{n}\left(\mathbb{C}^{m}\right)$ такой, что для $a=\sum_{\sigma \in S_{n}} p_{\sigma(1)} \otimes \cdots \otimes p_{\sigma(n)}$ имеет место формула

$$
a\left(x_{1}, \ldots, x_{n}\right)=\sum_{\sigma \in S_{n}} p_{\sigma(1)}\left(x_{1}\right) \otimes \cdots \otimes p_{\sigma(n)}\left(x_{n}\right)
$$

Возьмем в качестве $a$ элемент

$$
a=p \otimes 1 \otimes \cdots \otimes 1+1 \otimes p \otimes \cdots \otimes 1+\cdots+1 \otimes 1 \otimes \cdots \otimes p
$$


где $p \in \mathbb{C}\left[u_{1}, \ldots, u_{m}\right]$. Тогда

$$
\frac{1}{n !} \Phi_{n}(f)(p)=\sum_{k=1}^{n} p\left(x_{k}\right) .
$$

С другой стороны, используя линейность гомоморфизма $\Phi_{n}(f)$, его симметричность и форомулу (см. [3])

$$
\Phi_{n}(f)(p \otimes 1 \otimes \cdots \otimes 1)=f(p)(f(1)-1) \cdots(f(1)-(n-1)),
$$

мы получаем

$$
\frac{1}{n !} \Phi_{n}(f)(a)=f(p)
$$

так как $f(1)=n$, т.е. $f(p)=\sum_{k=1}^{n} p\left(x_{k}\right)$, и, следовательно, $f=\mathscr{E}\left(x_{1}, \ldots, x_{n}\right)$. Доказательство теоремы 11 закончено.

Теорема 12. Пусть $A$ - конечно порожденная коммутативная алгебра и V аффинное алгебраическое многообразие $\operatorname{Spec}(A)$. Тогда отображсение

$$
\begin{gathered}
\mathscr{E}: \operatorname{Sym}^{n}(V) \rightarrow \operatorname{Hom}(A, \mathbb{C}), \\
\mathscr{E}\left(f_{1}, \ldots, f_{n}\right)(a)=f_{1}(a)+\cdots+f_{n}(a),
\end{gathered}
$$

где $f_{k}: A \rightarrow \mathbb{C}, k=1, \ldots, n,-$ кольиевые гомоморфизмы, задает гомеоморфизм

$$
\mathscr{E}: \operatorname{Sym}^{n}(V) \rightarrow \Phi_{n}(A) .
$$

Подробное доказательство этого результата см. в [3].

Доказательство теоремы 8 на основе теоремы 9 проводится по той же схеме, что и доказательство теоремы 11. Дополнительно требуется воспользоваться лишь тем, что для компактного хаусдорфова пространства $X$ согласно теореме Стоуна-Вейерштрасса кольцо $C(X)^{\otimes n}$ всюду плотно в кольце $C\left(X^{n}\right)$ (детали см. в [16; утверждение 1.10.21]) и поэтому кольцо $S^{n}(C(X))$ всюду плотно в кольце $C\left(\operatorname{Sym}^{n}(X)\right)$. Это позволяет применить теорему Гельфанда-Колмогорова и по кольцевому гомоморфизму $\frac{1}{n !} \Phi_{n}(f): S^{n}(C(X)) \rightarrow \mathbb{C}$ получить точку пространства $\operatorname{Sym}^{n}(X)$.

\section{3. Свойства $n$-гомоморфизмов}

Как показьвают результаты предыдушего раздела, фробениусовы $n$-гомоморфизмы коммутативных алгебр имеют важные приложения. Таким образом возникла задача более детального изучения их свойств в этом специальном случае. При этом полезным оказалось другое описание определяюших уравнений для них, данное в $[4],[5]$.

Итак, пусть $A$ - коммутативная алгебра. Тогда:

1. Значение $\Phi_{n}(a, \ldots, a)=\Phi_{n}(f)(a \otimes \cdots \otimes a)$ для $a \in A$ равно детерминанту матрицы

$$
\left(\begin{array}{cccccc}
f(a) & 1 & 0 & 0 & \cdots & 0 \\
f\left(a^{2}\right) & f(a) & 2 & 0 & \cdots & 0 \\
f\left(a^{3}\right) & f\left(a^{2}\right) & f(a) & 3 & & 0 \\
\vdots & \vdots & \ddots & \ddots & \ddots & \\
\vdots & \vdots & & & f(a) & n-1 \\
f\left(a^{n}\right) & f\left(a^{n-1}\right) & \cdots & \cdots & f\left(a^{2}\right) & f(a)
\end{array}\right)
$$


2. Значение $\Phi_{n}(f)\left(a_{1}, \ldots, a_{n}\right)=\Phi_{n}(f)\left(a_{1} \otimes \cdots \otimes a_{n}\right)$ является полилинейной функцией переменных $a_{1}, \ldots, a_{n}$ и поэтому может быть получено стандартной процедурой поляризации из $\Phi_{n}(a, \ldots, a)$.

3. Представим перестановку $\sigma \in S_{n}$ в виде произведения непересекаюшихся циклов обшей длины $n$, скажем, $\sigma=\gamma_{1} \cdots \gamma_{r}$. Пусть $\gamma=\left(i_{1}, \ldots, i_{m}\right)$ - некоторьй цикл. Положим $f_{\gamma}\left(a_{1}, \ldots, a_{n}\right)=f\left(a_{i_{1}} \cdots a_{i_{m}}\right)$. Тогда имеет место формула

$$
\Phi_{n}(f)\left(a_{1}, \ldots, a_{n}\right)=\sum_{\sigma \in S_{n}} \varepsilon_{\sigma} f_{\gamma_{1}}\left(a_{1}, \ldots, a_{n}\right) \cdots f_{\gamma_{r}}\left(a_{1}, \ldots, a_{n}\right)
$$

где $\varepsilon_{\sigma}-$ знак перестановки $\sigma$.

Например,

$$
\begin{aligned}
\Phi_{3}(f)\left(a_{1}, a_{2}, a_{3}\right)= & f\left(a_{1}\right) f\left(a_{2}\right) f\left(a_{3}\right)-f\left(a_{1}\right) f\left(a_{2} a_{3}\right) \\
& -f\left(a_{2}\right) f\left(a_{1} a_{3}\right)-f\left(a_{3}\right) f\left(a_{1} a_{2}\right)+2 f\left(a_{1} a_{2} a_{3}\right) .
\end{aligned}
$$

Заметим, что формула (3.1) имеет место и в случае следовых гомоморфизмов $f$ некоммутативной алгебры $A$. В случае матричных алгебр $A$ и следовых гомоморфизмов $f$ эта формула появилась в [7].

Часто бьвает проще работать с формулой (3.1) в “диагональном” виде, а затем использовать поляризацию для получения общей формулы. Например, $\Phi_{3}(f)(a, a, a)=$ $s_{1}^{3}-3 s_{1} s_{2}+2 s_{3}$, где $s_{1}=f(a), s_{2}=f\left(a^{2}\right)$ и $s_{3}=f\left(a^{3}\right)$.

Мы применяем обозначение $s_{k}$, чтобы подчеркнуть связь с классической формулой Ньютона, выражающей элементарные симметрические функции $e_{n}=\sum t_{i_{1}} \cdots t_{i_{n}}$ коммутирующих переменных $t_{1}, t_{2}, \ldots$ (сумма берется по всем наборам $I_{n}=\left(i_{1}<\right.$ $\left.\left.\cdots<i_{n}\right)\right)$ как полиномы от степенных сумм $s_{k}=\sum t_{i}^{k}$ :

$$
n ! e_{n}=\operatorname{det}\left(\begin{array}{cccccc}
s_{1} & 1 & 0 & 0 & \cdots & 0 \\
s_{2} & s_{1} & 2 & 0 & \cdots & 0 \\
s_{3} & s_{2} & s_{1} & 3 & & 0 \\
\vdots & \vdots & \ddots & \ddots & \ddots & \\
\vdots & \vdots & & & s_{1} & n-1 \\
s_{n} & s_{n-1} & \cdots & \cdots & s_{2} & s_{1}
\end{array}\right)
$$

Опишем теперь развитие этой связи, обозначая через $F_{n}$ полином от переменных $s_{k}$, $k=1, \ldots, n$, которьй становится равным $\Phi_{n}(f)(a, \ldots, a)$ при подстановке $s_{k}=f\left(a^{k}\right)$. Непосредственно из определения полинома $F_{n}\left(s_{1}, \ldots, s_{n}\right)$ получаем формулу

$$
F_{n}=\sum_{\sigma \in S_{n}}\left(\prod_{k=1}^{n}\left((-1)^{k+1} s_{k}\right)^{m_{k}(\sigma)}\right)
$$

где $m_{k}(\sigma)$ - число циклов длины $k \geqslant 1$ в разложении перестановки $\sigma$ на произведение непересекаюшихся циклов. Далее, число элементов группы $S_{n}$ с $m_{k}$ циклами длины $k$ равно

$$
n ! / \prod_{k=1}^{n}\left(k^{m_{k}} m_{k} !\right)
$$


Следовательно,

$$
F_{n}=\sum_{\mathbf{m} \in \Pi(n)} \prod_{k=1}^{n}\left((-1)^{k+1} \frac{s_{k}}{k}\right)^{m_{k}}
$$

Здесь П $(n)$ - множество разбиений числа $n$ и $\mathbf{m}=\left(m_{1}, \ldots, m_{n}\right)$ - разбиение числа $n$, где $m_{k}-$ кратность числа $k$ в этом разбиении. Таким образом, $\sum_{k=1}^{n} k m_{k}=n$. Следовательно,

$$
F_{n} t^{n}=\sum_{\mathbf{m} \in \Pi(n)} \prod_{k=1}^{n}\left((-1)^{k+1} \frac{s_{k} t^{k}}{k}\right)^{m_{k}},
$$

и мы получаем, что имеет место

TEOPEMA 13.

$$
\sum_{n=0}^{\infty} F_{n} \frac{t^{n}}{n !}=\exp \left(\sum_{k=1}^{\infty}(-1)^{k+1} s_{k} \frac{t^{k}}{k}\right) .
$$

Из этого результата вытекает следующая рекурсия.

ПРЕДЛОЖЕНИЕ 14.

$$
F_{n}=(n-1) ! \sum_{k=1}^{n}(-1)^{k+1} s_{k} \frac{F_{n-k}}{(n-k) !} .
$$

ДоКАЗАТЕЛЬСТВо. Положим

$$
F(t)=\sum_{n=0}^{\infty} F_{n} \frac{t^{n}}{n !}
$$

Тогда согласно теореме 13 имеет место соотношение

$$
F^{\prime}(t)=F(t)\left(s_{1}-s_{2} t+s_{3} t^{2}-\cdots\right),
$$

из которого немедленно вытекает требуемая формула.

Дадим теперь характеризацию полиномов $F_{n}$, используя дифференциальные операторы.

Лемма 15. Пусть $d=\sum_{r=2}^{\infty} r s_{r-1} \frac{\partial}{\partial s_{r}}$. Тогда:
a) $\frac{\partial F_{n}}{\partial s_{1}}=n F_{n-1}$
б) $d F_{n}=-n(n-1) F_{n-1}$;
в) $\left[\frac{\partial}{\partial s_{k}}, d\right]=(k+1) \frac{\partial}{\partial s_{k+1}}$;
г) $\left(\operatorname{Ker} \frac{\partial}{\partial s_{1}}\right) \cap(\operatorname{Ker} d)$ состоит только из констант. 
ДокАЗАТЕЛЬСтво. а) Дифференцируя по $s_{1}$ правую часть соотношения из теоремы 13, получаем, что $\frac{\partial F(t)}{\partial s_{1}}=t F(t)$. Следовательно,

$$
\sum_{n=0}^{\infty} \frac{\partial F_{n}}{\partial s_{1}} \frac{t^{n}}{n !}=t \sum_{n=0}^{\infty} F_{n} \frac{t^{n}}{n !}
$$

Сравнивая коэффициенты при одинаковых степенях $t$, получаем результат а).

б) Применяя оператор $d$ к тому же соотношению, получаем

$$
d F(t)=F(t)\left[-s_{1} t^{2}+s_{2} t^{3}-s_{3} t^{4}+\cdots\right]=-t^{2} F^{\prime}(t)
$$

Опять сравнивая коэффиициенты при $t^{n}$, получаем

$$
\frac{d F_{n}}{n !}=-\frac{F_{n-1}}{(n-2) !}
$$

в) $d \frac{\partial}{\partial s_{k}}-\frac{\partial}{\partial s_{k}} d=\sum_{r=2}^{\infty} r s_{r-1} \frac{\partial}{\partial s_{r}} \frac{\partial}{\partial s_{k}}-\frac{\partial}{\partial s_{k}} \sum_{r=2}^{\infty} r s_{r-1} \frac{\partial}{\partial s_{k}}=(k+1) \frac{\partial}{\partial s_{k+1}}$.

г) Пусть $\frac{\partial f}{\partial s_{1}}=0$, тогда $f=f\left(s_{2}, s_{3}, \ldots\right)$. Если $f$ принадлежит пересечению ядер операторов $\frac{\partial}{\partial s_{1}}$ и $d$, то $\frac{\partial}{\partial s_{2}} f=0$ согласно утверждению в). Утверждение в) позволяет по индукции завершить доказательство результата г).

Из леммы 5 непосредственно вытекает следуюший результат.

ТЕорема 16. Последовательность полиномов $\left\{F_{n}\left(s_{1}, \ldots, s_{n}\right)\right\}$ полностью характеризуется следующими свойствами:

0) $F_{n}(0)=0, n=1,2, \ldots$,

1) $F_{0}=1$,

2) $\frac{\partial F_{n}}{\partial s_{1}}=n F_{n-1}$,

3) $d F_{n}=-n(n-1) F_{n-1}$

т.е. производящая функция $F(t)=F\left(t ; s_{1}, s_{2}, \ldots\right)$ является единственным решением системы уравнений

$$
\begin{aligned}
\frac{\partial}{\partial s_{1}} F(t) & =t F(t) \\
d F(t) & =-t^{2} \frac{\partial}{\partial t} F(t)
\end{aligned}
$$

при начальном условии $F\left(0 ; s_{1}, s_{2}, \ldots\right)=1$. 


\section{4. Алгебры Фробениуса}

Пусть $A$ - некоторая ассоциативная алгебра над $\mathbb{C}$. Линейное отображение $f: A \rightarrow \mathbb{C}$ называется следовым (англ. "trace-like"), если $f(a b)=f(b a)$ для любых $a$ и $b$ из $A$.

ОПрЕДЕЛЕнИЕ 5. Фробениусовой алгеброй $A$ назьвается алгебра $A$ вместе со следовым линейным отображением $f: A \rightarrow \mathbb{C}$ таким, что билинейная форма

$$
A \times A \rightarrow \mathbb{C}, \quad(a, b) \mapsto f(a b)
$$

является невырожденной.

Обозначим через $J(A)$ йорданову алгебру, которая аддитивно совпадает с $A$ и имеет умножение

$$
a \circ b=\frac{1}{2}(a b+b a) \text {. }
$$

Рассмотрим в $A$ некоторьй базис $\left\{e_{i}: i \in J\right\}$ и получим соответствующие ему структурные константы $a_{i j}^{k}$ (т.е. $\left.e_{i} e_{j}=\sum a_{i j}^{k} e_{k}\right)$. Тогда $a_{(i j)}^{k}=\frac{1}{2}\left(a_{i j}^{k}+a_{j i}^{k}\right)$ будут структурными константами йордановой алгебры $J(A)$ относительно того же базиса.

Теорема 17. Пусть $(A, f)$ - некоторая фробениусова алгебра. Тогда структурные константы йордановой алгебры $J(A)$ полностью определяются заданием гомоморфизмов $\Phi_{k}=\Phi_{k}(f), k=1,2 u 3$.

Результат, связанньй с теоремой 17 , при других предположениях был получен в [12]. Эта теорема включает утверждение, похожее на результат теоремы 2.8 из [3] и, следовательно, дает более сильньй результат.

С лЕДСТВИЕ 18. Для данного гомоморфизма $f: A \rightarrow \mathbb{C}$ линейные отображения $\Phi_{k}=\Phi_{k}(f): A^{\otimes n} \rightarrow \mathbb{C}, k \geqslant 4$, определяются отображсениями $\Phi_{1}, \Phi_{2}, \Phi_{3}$.

Из следствия 18 вытекает известньй результат [13] о том, что конечная группа полностью определяется своими $k$-характерами Фробениуса для $k=1,2,3$.

ДОКАЗАТЕЛЬСТВО ТЕОРЕМЫ 17 . По определению

$$
\Phi_{2}\left(e_{i}, e_{j}\right)=f\left(e_{i}\right) f\left(e_{j}\right)-f\left(e_{i} e_{j}\right) .
$$

Положим $R_{i j}=f\left(e_{i} e_{j}\right)$. Имеем

$$
R_{i j}=\sum_{r} a_{i j}^{r} f\left(e_{r}\right)=f\left(e_{i}\right) f\left(e_{j}\right)-\Phi_{2}\left(e_{i}, e_{j}\right)
$$

Аналогично,

$$
\begin{aligned}
\Phi_{3}\left(e_{i}, e_{j}, e_{k}\right)= & f\left(e_{i}\right) f\left(e_{j}\right) f\left(e_{k}\right)-f\left(e_{i}\right) \sum_{r} a_{j k}^{r} f\left(e_{r}\right) \\
& -f\left(e_{j}\right) \sum_{r} a_{i k}^{r} f\left(e_{r}\right)-f\left(e_{k}\right) \sum_{r} a_{i j}^{r} f\left(e_{r}\right) \\
& +\sum_{r, s}\left(a_{i j}^{r}+a_{j i}^{r}\right) a_{r k}^{s} f\left(e_{s}\right) .
\end{aligned}
$$


Следовательно,

$$
\begin{aligned}
\sum\left(a_{i j}^{r}+a_{j i}^{r}\right) R_{r k}= & f\left(e_{i}\right) R_{j k}+f\left(e_{j}\right) R_{i k}+f\left(e_{k}\right) R_{i j} \\
& -f\left(e_{i}\right) f\left(e_{j}\right) f\left(e_{k}\right)+\Phi_{3}\left(e_{i}, e_{j}, e_{k}\right) .
\end{aligned}
$$

Из определения фробениусовой алгебры следует, что матрица $R_{i j}=f\left(e_{i} e_{j}\right)=$ $\sum_{r} a_{i j}^{r} f\left(e_{r}\right)$ является симметричной и невырожденной. Рассматривая уравнение (4.2) при фиксированных $i, j$ как систему линейных уравнений относительно вектора $\left(a_{(i j)}^{r}\right.$, $r=1, \ldots, n)$, получаем, что эта система имеет единственное решение. Теорема доказана.

В случае коммутативной фробениусовой алгебры $A$ получается более явньй ответ.

Положим

$$
R_{i}=f\left(e_{i}\right), \quad R_{i j}=f\left(e_{i} e_{j}\right), \quad R_{i j k}=f\left(e_{i} e_{j} e_{k}\right) .
$$

В терминах значений гомоморфизмов $\Phi_{2}$ и $\Phi_{3}$ мы имеем

$$
\begin{aligned}
R_{i j} & =\Phi_{2}\left(e_{i}, e_{j}\right)-R_{i} R_{j}, \\
2 R_{i j k} & =\Phi_{3}\left(e_{i}, e_{j}, e_{k}\right)+R_{i} R_{j k}+R_{j} R_{i k}+R_{k} R_{i j}-R_{i} R_{j} R_{k} .
\end{aligned}
$$

Согласно определению фробениусовой алгебры матрица $R_{i j}$ обратима. Обозначим через $R^{i j}$ матрицу, обратную к $R_{i j}$. Прямые выгисления дают следующую явную формулу для структурных констант алгебры $A$.

ПРЕДЛОЖЕНИЕ 19. Структурные константы коммутативной фробениусовой алгебры задаются следующей формулой:

$$
a_{i j}^{k}=\sum_{m} R_{i j m} R^{m k}
$$

ДокАЗАТЕльСТво. Используя формулу

$$
R_{i j k}=f\left(e_{i} e_{j} e_{k}\right)=\sum_{n} f\left(a_{i j}^{n} e_{n} e_{k}\right)
$$

получаем

$$
\sum_{m} R_{i j m} R^{m k}=\sum_{m, n} a_{i j}^{n} R_{n m} R^{m k}=a_{i j}^{k}
$$

ДокАЗАТЕЛЬСТВо СЛЕДСТВИя 18. Нам достаточно показать, что для вычисления значений гомоморфизмов $\Phi_{k}$ необходимы структурные константы $a_{(i j)}^{k}$, а не $a_{i j}^{k}$. Используем индукцию.

В разложении $\Phi_{m}\left(e_{1}, \ldots, e_{m}\right)$ в терминах значений гомоморфизма $f$ единственное слагаемое, которое нельзя непосредственно выразить через $\Phi_{r}$ с $r<m$, есть

$$
\sum_{\sigma \in S_{m-1}} f\left(e_{1} e_{\sigma(2)} e_{\sigma(3)} \cdots e_{\sigma(m)}\right)=\frac{1}{m} \sum_{\sigma \in S_{m}} f\left(e_{\sigma(1)} e_{\sigma(2)} \cdots e_{\sigma(m)}\right) .
$$

Умножение в йордановой алгебре $J(A)$ может быть не ассоциативным, но ассоциатор выражается через коммутаторы, а именно:

$$
\begin{aligned}
4((a \circ b) \circ c-a \circ(b \circ c)) & =a b c+b a c+c a b+c b a-a b c-a c b-b c a-c b a \\
& =[b, a c]-[b, c a]=[b,[a, c]] .
\end{aligned}
$$


Так как гомоморфизм $f$ является следовым, то $f([a, b])=0$, и мы получаем, что

$$
f((a \circ b) \circ c)=f(a \circ(b \circ c)) .
$$

Следовательно, гомоморфизм $f$ на итерированных произведениях элементов алгебры $J(A)$ ведет себя так, как будто это ассоциативная алгебра. Таким образом,

$$
2^{m-1} \sum_{\sigma \in S_{m}} f\left(e_{\sigma(1)} \cdots e_{\sigma(m)}\right)=\sum_{\sigma \in S_{m}} f\left(e_{\sigma(1)} \circ \cdots \circ e_{\sigma(m)}\right) .
$$

В случае конечных групп получаем следующий результат.

Пусть $G$ - конечная группа с множеством элементов $\left\{g_{1}, \ldots, g_{n}\right\}$. Рассмотрим фробениусову алгебру $\mathbb{C} G$ со структурньм отображением $f=\frac{1}{n} \chi: \mathbb{C} G \rightarrow \mathbb{C}$, где $\chi-$ регулярное представление групшы $G$.

СлеДСТвиЕ 20. Линейнье отображения $\Phi_{1}=f, \Phi_{2}$ и $\Phi_{3}$ восстанавливают следующую структуру групповой алгебры $\mathbb{C} G$ :

1) $\Phi_{1}$ выцделяет единицу е группь $G$;

2) $\Phi_{2}$ задает операцию перехода $к$ обратному әлементу, т.е. выделяет паpы $\left(g_{i}, g_{j}\right)$ такие, что $g_{i} g_{j}=e$;

3) $\Phi_{1}, \Phi_{2}$ и $\Phi_{3}$ задают структурные константы йордановой алгебры $J(\mathbb{C} G)$.

ДокАЗАТЕльство. Регулярное представление $\chi$ принимает значение $n$ на единице $е$ группы $G$ и значение 0 на всех остальных элементах, откуда, очевидно, вытекает утверждение 1$)$, так как $\Phi_{1}=f=\frac{1}{n} \chi$. Далее, согласно (4.1)

$$
\Phi_{2}(h, g)= \begin{cases}-1, & \text { если } h g=e, h \neq e ; \\ 0 & \text { во всех остальных случаях. }\end{cases}
$$

Таким образом мы находим все пары взаимно обратных элементов. Перепишем полученный результат в терминах структурных констант. Упорядочим элементы группы $G$ так, чтобы $g_{1}=e$, и запишем $g_{i} g_{j}=\sum_{k=1}^{n} a_{i j}^{k} g_{k}$. В рассматриваемом случае $a_{i j}^{k}$ принимают значения 0 или 1 , причем $\sum_{k=1}^{n} a_{i j}^{k}=1$ для всех пар $(i, j)$. Утверждение 1$)$ означает, что

$$
a_{i 1}^{k}=a_{1 i}^{k}=\delta_{i, k} .
$$

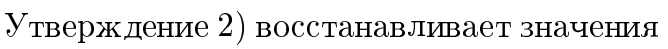

$$
a_{i j}^{1}=-\Phi_{2}\left(g_{i}, g_{j}\right), \text { если } i+j>2 .
$$

Выясним, какую информацию о структурных константах $a_{i j}^{k}$ несет отображение $\Phi_{3}$. Согласно (4.2)

$$
\Phi_{3}\left(g_{i}, g_{j}, g_{k}\right)=\sum_{r}\left(a_{i j}^{r}+a_{j i}^{r}\right) a_{r k}^{1}, \text { если } i+j+k>3 .
$$

Обратимая матрища $a_{r, k}^{1}$ нам полностью известна, что позволяет завершить доказательство. 
Теорема 21. Пусть $G$ - конечная группа и $\chi$-характер ее регулярного представления. Линейные отображения $\chi, \Phi_{2}(\chi)$ u $\Phi_{3}(\chi)$ определяют группу $G c$ точностью до изоморфизма, задаваемого отображсением $g \mapsto g^{-1}$.

ДокАЗАТЕльство. Используя следствие 20, немедленно получаем, что гомоморфизмы $\chi, \Phi_{2}(\chi)$ и $\Phi_{3}(\chi)$ задают умножение на множестве элементов группы $G$ с точностью до перестановки сомножителей.

Следуюшая лемма позволяет завершить доказательство этой теоремы.

ЛЕмма 22 (Р. Мэнсфильд [14]). Пусть $G$ - конечная группа, умножение в которой известно не точно, но так, что для каждой пары элементов $g, h \in G$ мь знаем множество значений $\{g h, h g\}$. Тогда мохсно восстановить множество функиий $\left\{m, m^{\mathrm{op}}: G \times G \rightarrow G\right\}$, где $m(x, y)=x y u m^{\mathrm{op}}(x, y)=y x$.

Доказательство этой леммы, приведенное в [14], представляет собой элементарный (но трюковый) перебор всех возможных случаев. Для полноты изложения мы приводим в приложении А (раздел 5) несколько упрошенное доказательство.

В [14] эта лемма была применена для доказательства результата Форманека и Сибли [8] о том, что групповой детерминант конечной групшы (впервые рассмотренньй еще Дедекиндом) определяет группу.

Групповой детерминант групшы $G$ определяется следующим образом.

Выберем взаимно однозначное соответствие между множеством элементов $\left\{g_{1}, \ldots\right.$, $\left.g_{n}\right\}$ группы $G$ и множеством коммутирующих переменных $\left\{x_{1}, \ldots, x_{n}\right\}$. Групповой детерминант - это определитель матрицы, полученной из таблицы умножения групшы заменой $g_{i} \rightarrow x_{i}$. Групповой детерминант является однородньм полиномом степени $n$ от $\left\{x_{1}, \ldots, x_{n}\right\}$ с целыми коэффициентами.

Зададим матрицу $M_{G}=\left(m_{i j}=x_{k}\right)$, где соответствие $(i, j) \rightarrow k$ задается правилом $g_{i} g_{j}^{-1}=g_{k}$. Ясно, что $M_{G}$ отличается от таблицы умножения порядком столбцов, так что $D_{G}= \pm \operatorname{det} M_{G}$. Отметим, что $\operatorname{det} M_{G}=x_{1}^{n}+\cdots$.

Рассмотрим $n$-мерное линейное пространство $\mathbb{C}^{n} \simeq \mathbb{C} G$ и правое регулярное представление групшы $G$ в базисе $\left\{g_{1}, \ldots, g_{n}\right\}$ :

$$
T: \mathbb{C}^{n} \rightarrow \mathbb{C}^{n}, \quad T(g) g_{k}=g_{k} g^{-1} .
$$

Обозначим через $T_{i}$ матрицу действия оператора $T\left(g_{i}\right)$ в этом базисе. Тогда

$$
M_{G}=\sum x_{i} T_{i}
$$

Рассмотрим теперь характер представления $T$

$$
\chi: \mathbb{C} G \rightarrow \mathbb{C}
$$

и продолжим его до линейного $\mathbb{C}\left[x_{1}, \ldots, x_{n}\right]$-гомоморфизма

$$
f: \mathbb{C} G\left[x_{1}, \ldots, x_{n}\right] \rightarrow \mathbb{C}\left[x_{1}, \ldots, x_{n}\right] .
$$

Лемма 23. Положим $a=\sum_{i=1}^{n} x_{i} g_{i} \in \mathbb{C} G\left[x_{1}, \ldots, x_{n}\right]$. Тогда

$$
\Phi_{n}(f)(a, \ldots, a)=D_{G} .
$$

Знаменитое разложение группового детерминанта на неприводимые множители, полученное Фробениусом в [10], получается немедленно из разложения регулярного представления $T$ на неприводимые: $n y c m b ~ \chi=\chi_{1}+\cdots+\chi_{q}-$ разложсение регулярного представления $T$ на неприводимые представления и $n_{i}$ - размерность представления с характером $\chi_{i}$, тогда

$$
D_{G}=\prod_{i=1}^{q} \Phi_{n_{i}}\left(\chi_{i}\right)^{n_{i}} .
$$




\section{5. Приложение А. Доказательство леммы Мэнсфильда}

Здесь мы приводим новое обешанное доказательство следующего результата.

ЛЕмма 24. Пусть $G$ - группа с умножением $(x, y) \rightarrow x y$. Тогда если *некоторое ассоциативное умножение на $G$, связанное с исходным умножением таким образом, что для каждой парь $x, y \in G$ произведение $x * y$ равно ху или $y x, \operatorname{mo~} x * y=x y$ для всех $x, y \in G$ или $x * y=y x$ для всех $x, y \in G$.

Переформулируем лемму следующим образом: пусть $A=\{(x, y): x * y=x y\}$ и $B=\{(x, y): x * y=y x\}$, тогда согласно условиям леммы $A \cup B=G \times G$, и надо доказать, что $A=G \times G$ или $B=G \times G$. Ясно, что множество $A \cap B$ симметрично.

Доказательство леммы опирается на упражнение 26 из $\$ 4$ в [1]. Для данной пары $x, y \in G$ мы имеем:

ФАKT 25. $x * y=y * x \Longleftrightarrow x y=y x$.

Импликация $x y=y x \Rightarrow x * y=y * x$ очевидна. В обратном направлении, пусть $x *$ $y=y * x$, тогда без ограничения обшности можно положить $x * y=x y$. Действительно, если это не так, то $y * x=x * y=y x$, и достаточно сделать замену $x$ и $y$.

Далее будем считать, что $x y \neq y x$, в противном случае доказывать нечего. Рассмотрим элемент $x * x * y=x^{2} * y$ и предположим, что он не равен $x^{2} y$. В то же время, в силу предыдуших соглашений $x * x * y=x * x y=x y x$ или $x^{2} y$. Таким образом, $y x^{2}=x y x$ и, следовательно, $y x=x y$. Полученное противоречие показывает, что $x * x * y=x^{2} y$.

Имеем $x * y * x * y=x y * x y=(x y)^{2}$. С другой стороны, по предположению элемент $x * y * x * y=x * x * y * y=x^{2} y * y$ равен $x^{2} y^{2}$ или $y x^{2} y$, т.е. $(x y)^{2}=x^{2} y^{2}$ или $(x y)^{2}=y x^{2} y$. И в том и в другом случае мы приходим к соотношению $x y=y x$, т.е. к противоречию.

СлЕДСТВИЕ 26. $x * y=x y \Longleftrightarrow y * x=y x$, m.e. множсество А симметрично.

ДокАЗАТЕльство. Пусть $x * y=x y$ и $y * x=x y$, тогда согласно факту 25 мы имеем $x y=y x$. Таким образом, $x * y=x y \Longrightarrow y * x=y x$. Обратная импликация следует по симметрии.

Так как оба множества $A$ и $A \cap B$ симметричны и $A \cup B=G \times G$, то мы немедленно получаем, что и $B$ симметричное множество. Таким образом, доказано, что имеет место

СлЕДСТВИЕ 27. $x * y=y x \Longleftrightarrow y * x=x y$.

ФАКТ 28. $x * y * x=x y x$ для всех $x, y \in G$.

ДокАЗАТЕЛЬСТВо. Пусть $x y=y x$, тогда $x * y * x=x y * x=x y x$ или $x^{2} y$. Требуемое равенство имеет место, так как $x y x=x^{2} y$. С другой стороны, пусть $x y \neq$ $y x$ и $x * y * x \neq x y x$. Тогда возможны следуюшие два случая.

a) $x * y=x y$ и $y * x=y x$. Тогда $x * y * x=x y * x=x^{2} y$ или $x y x$. По ассоциативности $x * y * x=x * y x=x y x$ или $y x^{2}$. Таким образом, $x * y * x=x^{2} y=y x^{2}$. Имеем: $x * x * y=x^{2} * y=x^{2} y=x * y * x$. Следовательно, $x * y=y * x$, что противоречит нашим предположениям.

б) $x * y=y x$ и $y * x=x y$. Опять мы имеем $x * y * x=y x * x=y x^{2}$ и $x * y * x=x * x y=$ $x^{2} y$. Таким образом, $x * x * y=x * y * x$ и $x * y=y * x$. Полученное противоречие завершает доказательство. 
Будем рассматривать теперь $G \times G$ как квадратную таблицу со строками $R_{x}=$ $\{(x, y): y \in G\}$.

ФАКт 29. Для любого $x \in G$ либо $R_{x} \subset A$, либо $R_{x} \subset B$.

ДокАЗАТЕльство. Допустим, что факт 29 не верен. Тогда сушествует такой элемент $x$, что $R_{x}$ пересекает оба множества $G \times G \backslash A$ и $G \times G \backslash B$, т.е. существуют такие $y, z \in G$, что $x * y=y x \neq x y=y * x$ и $x * z=x z \neq z x=z * x$. Покажем, что при этих предположениях $y x z=z x y$. Здесь возможны два случая.

а) $y * z \neq z * y$. Тогда, как показано вьше, $y z \neq z y$. Элемент $z * x * y$ равен $z x y$ или $y z x$. Этот элемент равен также $z * y x=z y x$ или $y x z$. Но $y z x \neq z y x, y z x \neq y x z$ и $z x y \neq z y x$, таким образом, $z * x * y=z x y=y x z$.

б) $y * z=z * y(=y z=z y)$. Покажем, что предположение $y x z \neq z x y$ приводит к противоречию. Рассмотрим элемент $z * x * y$. Он равен $z x * y=y z x$ или $z x y, \mathrm{a}$ также равен $z * y x=z y x$ или $y x z$. При наших предположениях, если $z x y \neq z y x$ и $y z x \neq y x z$, то мы получаем $z * x * y=y z x=z y x$. Рассмотрим элемент $x * z * y$. Он не равен $z * x * y=y z x$. Но он равен $x * y * z=x * y z=x y z$ или $y z x$. Следовательно, $x * z * y=x y z=x z y$. Наконец, рассмотрим элемент $z * y * x$. Он не равен $z * x * y=y z x$, но равен $y z * x=x y z$ или $y z x$. Следовательно, $z * y * x=x y z=x z y$. Таким образом, $x *(z * y)=(z * y) * x$, т.е. $x * z y=z y * x$, и поэтому $x z y=z y x$. Из этого мы получаем, что $z * x * y=x * z * y$, а это противоречит условию $x * z \neq z * x$.

Возьмем, наконец, элемент $x * z * x * y$. Согласно факту 2 он равен $x z x * y=x z x y$ или $y x z x$. С другой стороны, он равен $x z * y x=x z y x$ или $y x x z$. Опять надо рассматривать два случая.

а) $x * z * x * y=x z x y=x z y x$ или $y x x z$. При первом варианте получаем, что $x y=y x$, т.е. - противоречие. Второй вариант вместе с предыдушими вычислениями дает $y x x z=x z x y=x y x z$, что опять приводит к $x y=y x$.

б) $x * z * x * y=y x z x=x z y x$ или $y x x z$. Второй вариант дает $x z=z x$, т.е. противоречие. Таким образом, $x z y x=y x z x=z x y x$, но это также дает $x z=z x$. Это завершает доказательство факта 29.

Согласно факту 29 для каждого $x$ мы имеем $R_{x} \subset A$ или $R_{x} \subset B$. Предположим, что $R_{x} \subset A$, но $R_{x} \not \subset B$, и $R_{u} \subset B$, но $R_{u} \not \subset A$. Тогда $x * y=x y$ для всех $y$ и сушествует такое $z$, что $x z \neq z x$. Аналогично, $u * v=v u$ для всех $v$ и сушествует такое $w$, что $u w \neq w u$.

Рассмотрим элемент $x * u=x u$, он равен $u x=u * x$ и $R_{x u} \subset A$ или $B$. Предположим, что $R_{x u} \subset A$, тогда рассмотрим элемент $x * u * w=x u * w=x u w$, которьй должен быть равен также $x * w u=x w u$. Пришли к противоречию. Теперь предположим, что $R_{x u} \subset B$, и рассмотрим элемент $z * x * u=z * x u=x u z=u x z$, которьй также равен $z x * u=u z x$. Из этого вытекает, что $x z=z x-$ противоречие. Лемма Мэнсфильда доказана.

\section{6. Приложение В. Алгебра полисимметрических полиномов}

Пусть $n>1$. Напомним, что полином $p\left(x_{1}, \ldots, x_{n}\right)$, где $x_{k}=\left(x_{k 1}, \ldots, x_{k m}\right) \in \mathbb{C}^{m}$, называется полисимметрическим, если он инвариантен относительно всех перестановок множества аргументов $\left(x_{1}, \ldots, x_{n}\right)$. Алгебра полиномиальных функций на алгебраическом многообразии $\operatorname{Sym}^{n}\left(\mathbb{C}^{m}\right)$ канонически отождествляется с алгеброй полисимметрических полиномов, которую мы будем обозначать через $\mathscr{S}^{n}\left(\mathbb{C}^{m}\right)$. Для 
каждого набора неотрищательных целых чисел $\omega=\left(i_{1}, \ldots, i_{m}\right)$ введем полисимметрические полиномы Ньютона $p_{\omega}$ :

$$
p_{\omega}\left(x_{1}, \ldots, x_{n}\right)=\sum_{k=1}^{n} x_{1 k}^{i_{1}} \cdots x_{m k}^{i_{m}}
$$

видно, что при $m=1$ получаются классические полиномы Ньютона.

Положим $|\omega|=i_{1}+\cdots+i_{m}$. Пусть $\left\{z_{\omega}\right\}, \omega \in \mathbb{Z}_{\geqslant 0}^{m},-$ множество коммутируюших переменных, градуированных по правилу deg $z_{\omega}=|\omega|$. Рассмотрим градуированное кольцо полиномов $L=\mathbb{C}\left[z_{\omega}\right]$. Зададим в $L$ систему однородных полиномов $\left\{\mathscr{F}_{\omega_{1}, \ldots, \omega_{j}}\right\}, \operatorname{deg} \mathscr{F}_{\omega_{1}, \ldots, \omega_{j}}=\left|\omega_{1}\right|+\cdots+\left|\omega_{j}\right|$, по формулам "типа Фробениуса":

$$
\begin{aligned}
& \mathscr{F}_{\omega_{1}}=z_{\omega}, \quad \mathscr{F}_{\omega_{1}, \omega_{2}}=z_{\omega_{1}} z_{\omega_{2}}-z_{\omega_{1}+\omega_{2}} \quad \text { и далее по индукции: } \\
& \mathscr{F}_{\omega_{1}, \ldots, \omega_{j+1}}=z_{\omega_{1}} \mathscr{F}_{\omega_{2}, \ldots, \omega_{j+1}}-\mathscr{F}_{\omega_{1}+\omega_{2}, \omega_{3}, \ldots, \omega_{j+1}}-\ldots-\mathscr{F}_{\omega_{2}, \ldots, \omega_{j}, \omega_{1}+\omega_{j+1}} .
\end{aligned}
$$

Следуя схеме, приведенной вьше (см. раздел 3), нетрудно получить явное выражение полинома $\mathscr{F}_{\omega_{1}}, \ldots, \omega_{j}$. Для дальнейшего будет важным, что линейная часть полинома $\mathscr{F}_{\omega_{1}, \ldots, \omega_{j}}$ равна $(-1)^{j}(j-1) ! z_{\omega_{1}+\cdots+\omega_{j}}$.

Тот факт, что кольцо $L$ можно канонически отождествить с кольцом полиномов на линейном пространстве $\operatorname{Hom}\left(\mathbb{C}\left[u_{1}, \ldots, u_{m}\right], \mathbb{C}\right)$, позволяет, переходя к гомоморфизму колец полиномов, индуцированному отображением вычисления $\mathscr{E}$, получить из теоремы 11 следуюший результат.

ТЕОРЕМА 30. При данных $n$ и

$$
\mathscr{E}^{*}: L \rightarrow \mathscr{S} \mathscr{P}^{n}\left(\mathbb{C}^{m}\right)
$$

является әпиморфизмом.

Обозначим $\mathscr{S} \mathscr{Y}(m, n)$ ядро гомоморфизма $\mathscr{E}^{*}$. Тогда $\mathscr{S} \mathscr{Y}(m, n)-$ идеал в кольче $L$, порождденный полиномами $\mathscr{F}_{\omega_{1}, \ldots, \omega_{n+1}}$, при условии $\left|\omega_{1}\right| \cdots\left|\omega_{n+1}\right|>0$.

Рассмотрим подкольцо $L_{n} \subset L$, порожденное образуюшими $\left\{z_{\omega}\right\},|\omega|<n+1$.

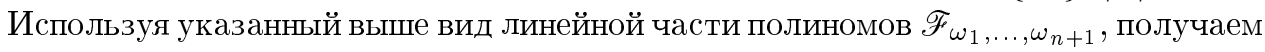

СлЕДСТВИЕ 31. Ограничение гомоморфизма $\mathscr{E}^{*}$ на $L_{n}$ дает эпиморфизм

$$
\mathscr{E}_{n}^{*}: L_{n} \rightarrow \mathscr{S} \mathscr{P}^{n}\left(\mathbb{C}^{m}\right)
$$

Обозначим через $\operatorname{Syz}(m, n)$ ядро гомоморфизма Ё*. Тогда

$$
\operatorname{Syz}(m, n)=\mathscr{S} \mathscr{Y} \mathscr{Z}(m, n) \cap L_{n} \text {. }
$$

По построению $L_{n}-$ кольцо полиномов на $\mathbb{C}^{N}$, где $N=\left(\begin{array}{c}n+m \\ n\end{array}\right)-1$. Получаем 
СлЕДСТВИЕ 32.

$$
\operatorname{Sym}^{n}\left(\mathbb{C}^{m}\right) \sim \operatorname{Spec}\left(L_{n} / \operatorname{Syz}(n, m)\right) \subset \mathbb{C}^{N} .
$$

Отметим, в заключение, что утверждение об эпиморфности гомоморфизма $\mathscr{E} *$ эквивалентно первой фундаментальной теореме классической теории инвариантов о том, что любой полисимметрический полином от $n$ векторных аргументов можно выразить (неоднозначно) в виде полинома от полисимметрических полиномов Ньютона $p_{\omega}$, $|\omega| \leqslant n$. Классический случай $m=1$ - единственньй, когда такое выражение однозначно.

\section{СПИСОК ЛИТЕРАТУРЫ}

[1] N. Bourbaki. Éléments de Mathématique. Algèbre. Chapitres 1-3. Paris: Hermann, 1970. (Пер. на рус. яз. (предыд. изд.): Н. Бурбаки. Алгебра, гл. 1-3. М.: Физматгиз, 1962.)

[2] В.М. Бухштабер, Е.Г. Рис. Конструктивное доказательство обобщенного изоморфизма Гельфанда // Функц. анализ и его прил. 2001. Т. 35. № 4. С. 20-25.

[3] V.M. Buchstaber, E. G. Rees. The Gelfand map and symmetric products // Selecta Math. (N.S.). 2002. V. 8. № 4. P. 523-535.

[4] V.M. Buchstaber, E. G. Rees. Multivalued groups, their representations and Hopf algebras // Transform. Groups. 1997. V. 2. № 4. P. 325-349.

[5] V.M. Buchstaber, E. G. Rees. Multivalued groups, $n$-Hopf algebras and $n$-ring homomorphisms // Lie Groups and Lie Algebras. Dordrecht: Kluwer, 1998. P. 85-107. (Math. Appl. V. 433.)

[6] В.М. Бухштабер, Е. Г. Рис. $k$-характеры Фробениуса и $n$-кольцевые гомоморфизмы // УМH. 1997. Т. 52. № 2. С. 159-160.

[7] E. Formanek. The Polynomial Identities and Invariants of $n \times n$ Matrices. Providence, RI: Amer. Math. Soc., 1991.

[8] E. Formanek, D. Sibley. The group determinant determines the group // Proc. Amer. Math. Soc. 1991. V. 112. № 3. P. 649-656.

[9] G. Frobenius. Über Gruppencharaktere // Sitzungsber. Preuß. Akad. Wiss. Berlin. 1896. P. 985-1021.

[10] G. Frobenius. Über die Primfaktoren der gruppendeterminante // Sitzungsber. Preuß. Akad. Wiss. Berlin. 1896. P. 1343-1382.

[11] И. М. Гельфанд, А. Н. Колмогоров. О колцах непрерывных функций на топологических пространствах // Докл. АН СССР. 1939. V. 22. № 1. Р. 11-15.

[12] H.- J. Hoehnke. Über komponierbare Formen und konkordante hyperkomplexe Größen // Math. Z. 1958. V. 70. P. 1-12.

[13] H.- J. Hoehnke, K. W. Johnson. The 1-, 2- and 3-characters determine a group // Bull. Amer. Math. Soc. (N.S.). 1992. V. 27. № 2. P. 243-245.

[14] R. Mansfield. A group determinant determines its group // Proc. Amer. Math. Soc. 1992. V. 116. № 4. P. 939-941.

[15] L. Nyssen. Pseudo-représentations // Math. Ann. 1996. V. 306. № 2. P. 257-283.

[16] T. W. Palmer. Banach Algebras and the General Theory of *-Algebras. V. 1: Algebras and Banach Algebras. Cambridge: Cambridge Univ. Press, 1994. (Encyclopedia Math. Appl. V. 49.)

[17] R. Rouquier. Caractérisation des caractères et pseudo-caractères // J. Algebra. 1996. V. 180. № 2. P. 571-586.

[18] R. L. Taylor. Galois representations associated to Siegel modular forms of low weight // Duke Math. J. 1991. V. 63. № 2. P. 281-332.

[19] A. Wiles. On ordinary $\lambda$-adic representations associated to modular forms // Invent. Math. 1988. V. 94. № 3. P. 529-573.

Математический институт им. В. А. Стеклова РАН;

University of Edinburgh, School of Mathematics

Поступила в редакцию

E-mail: buchstab@mendeleevo.ru; E.Rees@ed.ac.uk

15.01.2004 